\title{
Stability Analysis of Finite-Level Quantized Linear Control Systems
}

\author{
Carlos E. de Souza
}

\author{
Daniel F. Coutinho
}

Minyue $\mathrm{Fu}$

\begin{abstract}
In this paper we investigate the stability of discrete-time linear time-invariant systems subject to finite-level logarithmic quantized feedback. Both state feedback and output feedback are considered. We develop an LMI approach to estimate, for a given controller and a given finite-level quantizer, a set of admissible initial states and an associated attractor set in a neighborhood of the origin such that all state trajectories starting in the first set will converge to the attractor in a finite time and will never leave it. Furthermore, when these two such sets are a priori specified, we develop sufficient conditions for designing a suitable state or output feedback controller, along with a finite-level logarithmic quantizer.
\end{abstract}

\section{INTRODUCTION}

Motivated by the huge interest in network-based feedback control systems, the study of quantization errors has become an important area of research. There are many situations in which quantization errors may arise and its effects cannot be neglected at the cost of poor closed-loop performance and even the lost of stability.

Early results on quantized feedback concentrate on analyzing the effects of quantization and more recently mitigating them [1]-[3]. Nowadays, networked control systems are the most popular examples of systems subject to quantization. In such systems, the plant and the control elements (sensor, controller and actuator) are interconnected through digital communication channels with a finite bandwidth. Since in networked systems the control elements share the same communication link, a natural issue for such systems is to minimize the amount of information needed to be transmitted while achieving a certain closed-loop performance. Over the past few years a significant number of works has focused on this topic; see, e.g. [4]-[11].

Research on quantized feedback systems can be divided into static and dynamic quantizers. A static quantizer is a memoryless nonlinear function and the dynamic one uses memory to improve the performance at the cost of higher complexity. To overcome the complexity problem, several researchers have employed a static quantizer together with a dynamic scaling method in which a scaling factor is

This work was supported by $\mathrm{CNPq}$, Brazil, under grants $47.1739 / 06-5$, 45.4495/07-2, 20.0018/08-6, 30.2317/02-3/PQ and 30.2741/05-4/PQ, and by the ARC Centre for Complex Dynamic Systems and Control, Australia.

C.E. de Souza is with the Department of Systems and Control, Laboratório Nacional de Computação Científica (LNCC/MCT), Petrópolis, RJ 25651-075, Brazil (csouza@lncc.br)

D.F. Coutinho is with the Laboratoire d'Automatique, Faculté Polytechnique de Mons, Boulevard Dolez 31, B-7000 Mons, Belgium, and on leave from GACS, FENG-PUCRS, Brazil (dcoutinho@pucrs.br)

$\mathrm{M} . \mathrm{Fu}$ is with the School of Electrical Engineering and Computer Science, University of Newcastle, Callaghan, NSW 2308, Australia. (minyue.fu@newcastle.edu.au) dynamically adjusted to achieve (semi-) global asymptotic stability [5], [10], [12].

For static quantizers, it has been demonstrated in [7] that the coarsest quantization density for quadratic stabilization of discrete-time single-input single-output (SISO) linear timeinvariant (LTI) systems using quantized state feedback is achieved by using a logarithmic quantizer. This result was extended in [11], [13] in several directions (such as, MIMO systems, output feedback with quadratic or $H_{\infty}$ performance, and systems with input and output logarithmic quantizers) using the sector bound approach. Notice that in the two later works the logarithmic quantizer has an infinite number of quantization levels, which is not practically implementable. To address the issue of finite-level quantization, using the sector bound approach [12] introduced a dynamic scaling method for the logarithmic quantizer.

In this paper, we extend the sector bound approach [11] to handle finite-level logarithmic quantizers without the use of dynamic scaling. The motivation for employing logarithmic quantizers is that they bring in several advantages, such as a convex characterization of quadratic stabilization and the explicit coarsest quantization density formulae. More importantly, logarithmic quantization gives high-resolution quantization when the input is small but low-resolution quantization when the input is large, resulting in a roughly constant relative error, which is naturally required in many applications. We consider discrete-time SISO linear timeinvariant systems with a given finite-level logarithmically quantized feedback and for a given state or output feedback controller. For these systems, we develop an LMI approach to estimate a set of admissible initial states and an invariant set in the neighborhood of the origin for which all state trajectories starting in the first set will be attracted to in finite time and will never leave it. Furthermore, in the case where these two such sets are a priori specified, we provide a procedure to design either a state feedback or an output feedback controller, as well as a finite-level logarithmic quantizer to guarantee the aforementioned convergence property. A numerical example demonstrates the potentials of the proposed approach and shows that it can be used as a tool to design finite-level quantized feedback controllers.

Notation. Our notation is quite standard. For a real matrix $S, S^{\prime}$ denotes its transpose and $S>0(S \geq 0)$ means that $S$ is symmetric and positive definite (nonnegative definite). For two sets $\mathcal{A}$ and $\mathcal{B}$ such that $\mathcal{B} \subset \mathcal{A}$, the notation $\mathcal{A} \backslash \mathcal{B}$ stands for $\mathcal{A}$ excluded $\mathcal{B}$. 


\section{Problem Statement}

Consider the following SISO linear system:

$$
\left\{\begin{aligned}
x(k+1) & =A x(k)+B u(k) \\
y(k) & =C x(k)
\end{aligned}\right.
$$

where $A \in \mathbb{R}^{n \times n}, B \in \mathbb{R}^{n}, C^{\prime} \in \mathbb{R}^{n}, x$ is the state, $u$ is the control signal and $y$ is the measurement.

The above system will be controlled by either a quantized state feedback

$$
r(k)=K x(k), \quad u(k)=Q(r(k))
$$

or a dynamic output feedback controller of the form

$$
\left\{\begin{aligned}
\xi(k+1) & =A_{c} \xi(k)+B_{c} v(k) \\
w(k) & =C_{c} \xi(k)+D_{c} v(k)
\end{aligned}\right.
$$

where $K^{\prime} \in \mathbb{R}^{n}$ is the state feedback gain, $Q(\cdot)$ is a static symmetric quantizer to be specified later, $A_{c} \in \mathbb{R}^{n_{c} \times n_{c}}, B_{c} \in$ $\mathbb{R}^{n_{c}}, C_{c}^{\prime} \in \mathbb{R}^{n_{c}}$ and $D_{c} \in \mathbb{R}$ are the matrices of the output feedback controller, $\xi$ is its state, and $v$ and $w$ are related to $y$ and $u$, respectively, as specified below.

Without loss of generality, we assume that $(A, B, C)$ and $\left(A_{c}, B_{c}, C_{c}, D_{c}\right)$ are minimal realizations.

In the output feedback case we consider two possible configurations [11] involving the system (1), controller (3) and a quantizer $Q(\cdot)$ as follows:

- Configuration I. The measurement is quantized but the control signal is not. In this case, $v(k)=Q(y(k))$ and $u(k)=w(k)$.

- Configuration II. The control signal is quantized but the measurement is not. In this case, $u(k)=Q(w(k))$ and $v(k)=y(k)$.

It is assumed that the quantizer $Q(\cdot)$ has a logarithmic law with quantization levels given by

$\mathcal{V}=\left\{ \pm m_{i}: m_{i}=\rho^{i} \mu, i=0,1,2, \cdots, N-1\right\} \cup\{0\}, \rho \in(0,1)$

where $N$ is the number of positive quantization levels and $\mu>0$ is the largest admissible level. Note that a small (large) $\rho$ implies coarse (dense) quantization. As an abuse of terminology, $\rho$ will be called the quantization density.

In this paper, we investigate the closed-loop stability of system (1) with either the state-feedback law in (2) or the output feedback controller in (3) in Configurations I or II, and a logarithmic quantizer with a finite alphabet following the constructive law defined below

$$
Q(v)= \begin{cases}\mu, & \text { if } v>\frac{\mu}{(1-\delta)}, \quad \mu>0 \\ \rho^{i} \mu, & \text { if } \frac{\rho^{i} \mu}{(1+\delta)}<v \leq \frac{\rho^{i} \mu}{(1-\delta)}, \\ \quad & i=0,1, \ldots, N-1 \\ 0, & \text { if } 0 \leq v \leq \frac{\rho^{N-1} \mu}{(1+\delta)} \\ -Q(-v), & \text { if } v<0\end{cases}
$$

where

$$
\delta=\frac{1-\rho}{1+\rho} .
$$

\section{Previous Results}

This section reviews two results proposed in [11], where the quadratic stabilization of linear feedback systems with a logarithmic quantizer with an infinite number of levels is solved using the sector bound approach and $H_{\infty}$ optimization. Let the logarithmic quantizer $\bar{Q}(\cdot)$ with an infinite number of levels as shown in Fig. 1 and defined by:

$$
\bar{Q}(v)=\left\{\begin{array}{lc}
\rho^{i} \mu, & \text { if } \frac{\rho^{i} \mu}{(1+\delta)}<v \leq \frac{\rho^{i} \mu}{(1-\delta)}, \\
& i=0,1, \ldots \\
0, & \text { if } v=0 \\
-\bar{Q}(-v), & \text { if } v<0
\end{array}\right.
$$

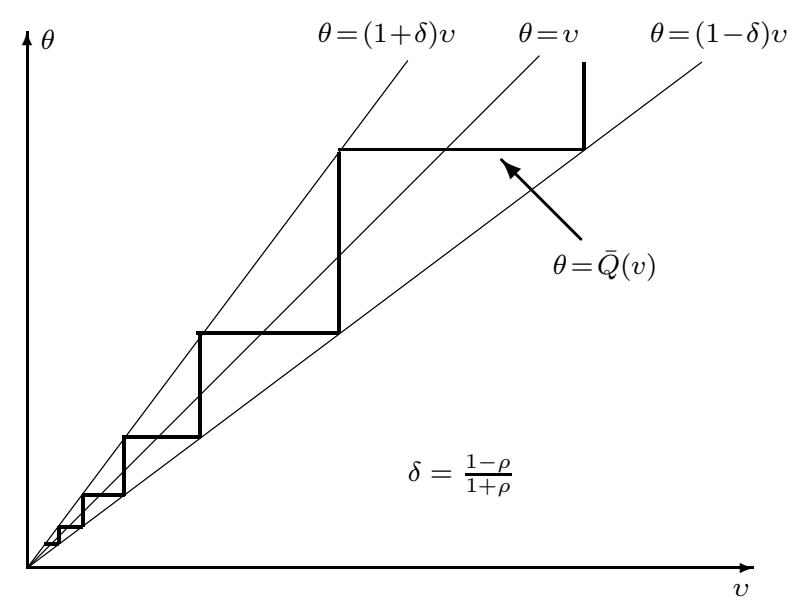

Fig. 1. Logarithmic quantizer with an infinite number of levels.

Notice from Fig. 1 that the quantizer $\bar{Q}(\cdot)$ can be bounded by a sector $(1+\Delta) v$, where $\Delta \in[-\delta, \delta]$.

If we consider the system (1) with the controller of either (2) or (3) in Configurations I or II, we get from [11] and [13] the following results.

Theorem 3.1: Consider the system (1). For a given quantization density $\rho$, this system is quadratically stabilizable via a quantized state feedback controller (2) with $Q(\cdot) \equiv \bar{Q}(\cdot)$, if and only if the following auxiliary system:

$$
x(k+1)=A x(k)+B(1+\Delta) r(k), \quad|\Delta| \leq \delta
$$

is quadratically stabilizable with $r(k)=K x(k)$, where $\delta$ and $\rho$ are related by (5). Moreover, the largest sector bound $\delta_{\text {sup }}$ for quadratic stabilization, which provides the smallest quantization density $\rho_{\text {inf }}$, is given by

where

$$
\delta_{\text {sup }}=\frac{1}{\inf _{K}\left\|G_{K}(z)\right\|_{\infty}}
$$

$$
G_{K}(z)=K(z I-A-B K)^{-1} B .
$$

Theorem 3.2: Let the system (1) and a quantizer $\bar{Q}(\cdot)$ in either Configurations I or II. For a given quantization density $\rho$, this system is quadratically stabilizable via an output feedback controller (3) if and only if the system

$$
\left\{\begin{aligned}
x(k+1) & =A x(k)+B w(k) \\
v(k) & =(1+\Delta) C x(k), \quad|\Delta| \leq \delta
\end{aligned}\right.
$$


in the case of Configuration I, or the system

$$
\left\{\begin{aligned}
x(k+1) & =A x(k)+B(1+\Delta) w(k) \\
v(k) & =C x(k), \quad|\Delta| \leq \delta
\end{aligned}\right.
$$

in the case of Configuration II, is quadratically stabilizable via a controller (3), where $\delta$ and $\rho$ are related by (5). Moreover, for both configurations, the largest sector bound $\delta_{\text {sup }}$ for quadratic stabilization, which provides the smallest quantization density $\rho_{\text {inf }}$, is given by

where

$$
\delta_{\mathrm{sup}}=\frac{1}{\inf _{A_{c}, B_{c}, C_{c}, D_{c}}\|\bar{G}(z)\|_{\infty}}
$$

$$
\begin{gathered}
\bar{G}(z)=G(z) H(z)[1-G(z) H(z)]^{-1}, \\
G(z)=C(z I-A)^{-1} B, \quad H(z)=C_{c}\left(z I-A_{c}\right)^{-1} B_{c}+D_{c} .
\end{gathered}
$$

Remark 3.1: Finding the optimal $K$ and $\left(A_{c}, B_{c}, C_{c}, D_{c}\right)$ of Theorems 3.1 and 3.2, respectively, is a convex $H_{\infty}$ optimization problem. Thus, these parameters can be readily determined by means of the LMI framework [14].

\section{Stability Analysis}

The results of Section III apply to quantized feedback systems for which the quantizer has an infinite number of quantization levels. When dealing with finite-level quantizers, in general, we cannot assure that the state trajectory will converge to the state-space origin (the equilibrium point under analysis). In the sequel we shall derive LMI conditions to ensure the convergence in finite time of the state trajectory to a small invariant neighborhood of the origin.

\section{A. General Setup}

First, we introduce an auxiliary system which encompasses the closed-loop system for the three feedback control laws with the finite-level quantizer in (4) under analysis, namely the state-feedback controller (2) and the output feedback controller (3) in either Configuration I or II. To this end, we define the following system:

$$
\left\{\begin{aligned}
\zeta(k+1) & =A_{i} \zeta(k)+B_{i} Q(v(k)) \\
v(k) & =C_{i} \zeta(k), \quad i=1,2,3
\end{aligned}\right.
$$

where $Q(\cdot)$ is the quantizer function as defined in (4) and the index $i$ is related to the feedback control under consideration. More specifically, $i=1$ refers to state feedback, $i=2$ is for output feedback in Configuration I, and $i=3$ refers to output feedback in Configuration II. From straightforward algebraic manipulations, we obtain the following results:

$$
\begin{gathered}
\zeta=x, \text { for } i=1, \quad \zeta=\left[\begin{array}{ll}
x^{\prime} & \xi^{\prime}
\end{array}\right]^{\prime}, \quad \text { for } i=2,3 \\
A_{1}=A, \quad B_{1}=B, \quad C_{1}=K \\
A_{2}=\left[\begin{array}{cc}
A & B C_{c} \\
0 & A_{c}
\end{array}\right], \quad A_{3}=\left[\begin{array}{cc}
A & 0 \\
B_{c} C & A_{c}
\end{array}\right], \quad B_{2}=\left[\begin{array}{c}
B D_{c} \\
B_{c}
\end{array}\right] \\
B_{3}=\left[\begin{array}{ll}
B^{\prime} & 0
\end{array}\right]^{\prime}, \quad C_{2}=\left[\begin{array}{ll}
C & 0
\end{array}\right], \quad C_{3}=\left[\begin{array}{ll}
D_{c} C & C_{c}
\end{array}\right] .
\end{gathered}
$$

In connection with the closed-loop system (14) and the finite-level quantizer (4), let the following sets:

$$
\begin{gathered}
\mathcal{B}=\left\{\zeta \in \mathbb{R}^{n_{i}}:\left|C_{i} \zeta\right| \leq \mu /(1-\delta)\right\} \\
\mathcal{C}=\left\{\zeta \in \mathbb{R}^{n_{i}}:\left|C_{i} \zeta\right| \leq \varepsilon\right\}, \quad \varepsilon=\rho^{N-1} \mu /(1+\delta)
\end{gathered}
$$

where $\delta$ and $\mu$ are as in (4), $i=1,2$ or 3 , depending on the feedback being considered, whereas $n_{1}=n$ and $n_{2}=n_{3}=$ $n+n_{c}$. The sets $\mathcal{B}$ and $\mathcal{C}$ are related to respectively the largest and smallest quantization levels. These sets are unbounded along the directions of the vectors of an orthogonal basis of the null space of $C_{i}$ and bounded by two hyperplanes orthogonal to $C_{i}^{\prime}$ and symmetric with respect to origin. The distance between these hyperplanes is $2 \mu(1-\delta)^{-1} / \sqrt{C_{i} C_{i}^{\prime}}$ for $\mathcal{B}$ and $2 \varepsilon / \sqrt{C_{i} C_{i}^{\prime}}$ for $\mathcal{C}$.

Note that when the state $\zeta$ of system (14) lies in $\mathcal{C}$, $Q\left(C_{i} \zeta\right)=0$, then the input signal to the latter system is zero. Thus, in general, the trajectory of $\zeta$ will not converge to the origin and hence quadratic stability will not hold. To handle this situation, and inspired by the notion of practical stability used in [7], in the sequel we will introduce the notion of stability adopted in this paper. Let the Lyapunov functions

$$
V(\zeta)=\zeta^{\prime} P \zeta, \quad V_{a}(\zeta)=\zeta^{\prime} P_{a} \zeta, \quad P>0, \quad P_{a}>0
$$

where $\zeta$ is as in (15), and the sets:

$$
\begin{gathered}
\mathcal{D}=\left\{\zeta \in \mathbb{R}^{n_{i}}: V(\zeta) \leq 1\right\}, \quad \mathcal{A}=\left\{\zeta \in \mathbb{R}^{n_{i}}: V_{a}(\zeta) \leq 1\right\} \\
\mathcal{C}_{p}=\left\{\zeta \in \mathcal{C}: D V_{a}(\zeta) \geq 0\right\}
\end{gathered}
$$

where the notation $D f(\zeta(k))$, for a real function $f(\cdot)$, is defined by $D f(\zeta(k)):=f(\zeta(k+1))-f(\zeta(k))$.

Definition 4.1: Consider the closed-loop system (14) with either the state feedback (2) or the output feedback (3) in Configuration I or II. This system is widely quadratically stable, if there exists Lyapunov functions $V(\zeta)$ and $V_{a}(\zeta)$ as above such that the following conditions hold:

$$
\begin{aligned}
& \mathcal{A} \subset \mathcal{D}, \quad \mathcal{D} \subset \mathcal{B} \\
& D V(\zeta)<0, \forall \zeta \in \mathcal{D} \backslash \mathcal{C} \\
& D V_{a}(\zeta)<0, \forall \zeta \in \mathcal{A} \backslash \mathcal{C}_{p} \\
& \zeta(k+1) \in \mathcal{A} \text { whenever } \zeta(k) \in \mathcal{C}_{p} .
\end{aligned}
$$

Definition 4.1 implies that for any initial condition in $\mathcal{D}$, the state trajectory of system (14) will enter $\mathcal{A}$ in finite time and will remain in this set. Thus, $\mathcal{A}$ is an attractor of $\mathcal{D}$ and the latter set will be referred to as the set of admissible initial states (or conditions). Note that Definition 4.1 allows for different shapes for $\mathcal{D}$ and $\mathcal{A}$, which is a desired feature due to the shape of $\mathcal{B}$. However, one can constrain $\mathcal{A}$ to have the same shape as $\mathcal{D}$ by setting $P_{a}=\beta P$, with $\beta>1$. Observe that some of the features of Definition 4.1 are similar to those of the practical stability in [7].

\section{B. Main Results}

First, considering (14), condition $D V(\zeta)<0$ is given by

$$
\left[\begin{array}{c}
\zeta \\
Q(v)
\end{array}\right]^{\prime}\left[\begin{array}{cc}
A_{i}^{\prime} P A_{i}-P & A_{i}^{\prime} P B_{i} \\
B_{i}^{\prime} P A_{i} & B_{i}^{\prime} P B_{i}
\end{array}\right]\left[\begin{array}{c}
\zeta \\
Q(v)
\end{array}\right]<0
$$

where $v$ is as in (14). Also, notice that for all $\zeta \in \mathcal{B} \backslash \mathcal{C}$, $Q(v)$ satisfies the following sector bound condition [11]:

$$
(Q(v)-(1-\delta) v)^{\prime}(Q(v)-(1+\delta) v) \leq 0
$$


Thus, condition (25) is satisfied iff (28) holds subject to (29). By applying the $\mathcal{S}$-procedure [15] the latter holds iff

$$
\eta^{\prime}\left[\begin{array}{cc}
A_{i}^{\prime} P A_{i}-P-\tau_{1}\left(1-\delta^{2}\right) C_{i}^{\prime} C_{i} & A_{i}^{\prime} P B_{i}+\tau_{1} C_{i}^{\prime} \\
B_{i}^{\prime} P A_{i}+\tau_{1} C_{i} & B_{i}^{\prime} P B_{i}-\tau_{1}
\end{array}\right] \eta<0
$$

where $\eta=\left[\begin{array}{ll}\zeta^{\prime} & Q(v)^{\prime}\end{array}\right]^{\prime}$ and $\tau_{1}>0$ is a multiplier to be found introduced by the $\mathcal{S}$-procedure.

Observe that condition (30) with $P$ and $\tau_{1}$ replaced by $P_{a}$ and $\tau_{2}$, respectively, ensures that $D V_{a}(\zeta)<0, \forall \zeta \in \mathcal{B} \backslash \mathcal{C}$. This together with (24) and considering the definition of the set $\mathcal{C}_{p}$, will ensure the feasibility of (26).

Theorem 4.1: Let $Q(\cdot)$ be a finite-level quantizer as defined in (4), where $\mu, \rho$ and $N$ are given, and consider the system (1) with either a given state feedback controller (2) or an output feedback controller (3) in Configuration I or II. The resulting closed-loop system (14) is widely quadratically stable if there exist matrices $P>0$ and $P_{a}>0$, and positive scalars $\tau_{1}, \cdots, \tau_{4}$ satisfying the following inequalities:

$$
\begin{gathered}
P_{a}-P>0 \\
P-(1-\delta)^{2} \mu^{-2} C_{i}^{\prime} C_{i}>0 \\
{\left[\begin{array}{cc}
A_{i}^{\prime} P A_{i}-P-\tau_{1}\left(1-\delta^{2}\right) C_{i}^{\prime} C_{i} & A_{i}^{\prime} P B_{i}+\tau_{1} C_{i}^{\prime} \\
B_{i}^{\prime} P A_{i}+\tau_{1} C_{i} & B_{i}^{\prime} P B_{i}-\tau_{1}
\end{array}\right]<0} \\
{\left[\begin{array}{cc}
A_{i}^{\prime} P_{a} A_{i}-P_{a}-\tau_{2}\left(1-\delta^{2}\right) C_{i}^{\prime} C_{i} & A_{i}^{\prime} P_{a} B_{i}+\tau_{2} C_{i}^{\prime} \\
B_{i}^{\prime} P_{a} A_{i}+\tau_{2} C_{i} & B_{i}^{\prime} P_{a} B_{i}-\tau_{2}
\end{array}\right]<0} \\
\tau_{3}-\tau_{4} \geq 0 \\
P_{a}-\left(1+\tau_{3}\right) A_{i}^{\prime} P_{a} A_{i}+\tau_{4} \varepsilon^{-2} C_{i}^{\prime} C_{i} \geq 0
\end{gathered}
$$

where $\delta$ is related to $\rho$ by (5) and $\varepsilon$ is as in (20). Moreover, the set $\mathcal{D}$ of admissible initial states and its attractor $\mathcal{A}$ are given be (22).

Proof. Firstly, in view of (19) and (22), the inequalities (31) and (32) ensure that $\mathcal{A} \subset \mathcal{D}$ and $\mathcal{D} \subset \mathcal{B}$, respectively.

Next, (33) guarantees that (30) holds, implying that condition (25) is satisfied. Similarly, (34) together with (24) and the definition of set $\mathcal{C}_{p}$ ensures that condition (26) holds.

Adding (35) to (36) post-multiplied by $\phi \in \mathbb{R}^{n_{i}}$ and premultiplied by $\phi^{\prime}$, we get

$$
\begin{aligned}
& \left(1-\phi^{\prime} A_{i}^{\prime} P_{a} A_{i} \phi\right)-\tau_{3}^{-1} \phi^{\prime}\left(A_{i}^{\prime} P_{a} A_{i}-P_{a}\right) \phi \\
& \quad-\tau_{3}^{-1} \tau_{4}\left(1-\varepsilon^{-2} \phi^{\prime} C_{i}^{\prime} C_{i} \phi\right) \geq 0, \forall \phi \in \mathbb{R}^{n_{i}} .
\end{aligned}
$$

By the $S$-procedure, the latter inequality implies that

$$
\left\{\begin{array}{r}
\phi^{\prime} A_{i}^{\prime} P_{a} A_{i} \phi \leq 1, \forall \phi \in \mathbb{R}^{n_{i}}: \varepsilon^{-2} \phi^{\prime} C_{i}^{\prime} C_{i} \phi \leq 1, \\
\phi^{\prime}\left(A_{i}^{\prime} P_{a} A_{i}-P_{a}\right) \phi \geq 0 .
\end{array}\right.
$$

Note that the second inequality of (37) is equivalent to $\phi \in \mathcal{C}$. With $\phi=\zeta(k)$ as in (14), and considering that for $\zeta(k) \in \mathcal{C}$ the input signal $Q(v)$ of (14) is zero, then (37) leads to

$$
\left\{\begin{array}{l}
\zeta(k+1)^{\prime} P_{a} \zeta(k+1) \leq 1, \\
\quad \forall \zeta(k) \in \mathcal{C}: \zeta(k+1)^{\prime} P_{a} \zeta(k+1)-\zeta(k)^{\prime} P_{a} \zeta(k) \geq 0
\end{array}\right.
$$

which ensures that condition (27) is satisfied. Hence, we conclude that system (14) is widely quadratically stable.
Remark 4.1: Notice that in Theorem 4.1 the controller and the quantizer $Q(\cdot)$ are considered to be known. A possible way to determine a controller (state or output feedback) is to employ the design of either Theorem 3.1 or 3.2 for logarithmic quantizers with an infinite number of quantization levels, and choose the quantization density $\rho$ of the finite-level quantized such that $\rho \geq \rho_{\text {inf }}$, where $\rho_{\text {inf }}$ is the smallest quantization density given by these theorems. The maximum quantization level $\mu$ and the zero-level quantization error $\varepsilon=(1+\delta)^{-1} \rho^{N-1} \mu$ are then chosen by the designer. Observe that for a given $\mu$, Theorem 4.1 can be used to determine the maximum admissible zero-level quantization error, which gives the smallest admissible $N$. This can be achieved by searching for the largest value of $\varepsilon>0$ such that the inequalities (31)-(36) of Theorem 4.1 are feasible.

Remark 4.2: Observe that (36) is not jointly convex in $\tau_{3}$ and $P_{a}$. However, for a given $\tau_{3}$ the inequalities (31)(36) become LMIs. Thus, a direct approach to solve these inequalities is to search for the parameter $\tau_{3}>0$. A line search seems to be an appropriate way to optimize $\tau_{3}$.

In general, it is desirable to find the set $\mathcal{D}$ of maximum size, in the sense of its volume, or the smallest $\mathcal{A}$. Since $\mathcal{D}$ is an ellipsoid, one approach to maximize its size is to minimize $\operatorname{Trace}(P)$. The motivation for this is that $n_{i}(\operatorname{Trace}(P))^{-1} \leq$ $\operatorname{Trace}\left(P^{-1}\right), P \in \mathbb{R}^{n_{i} \times n_{i}}$, and $\operatorname{Trace}\left(P^{-1}\right)$ is the sum of the squared semi-axis lengths of the ellipsoid $\mathcal{D}$. Similarly, an approach to minimize the size of $\mathcal{A}$ is to maximize $\operatorname{Trace}\left(P_{a}\right)$. In light of the latter arguments, the size of the set $\mathcal{D}$ of Theorem 4.1 can be maximized by solving the following optimization problem:

$$
\begin{aligned}
& \min _{\gamma_{1}, P, P_{a}, \tau_{1}, \cdots, \tau_{4}} \gamma_{1}, \quad \text { subject to (31)-(36) and } \\
& \quad P>0, \quad \tau_{i}>0, i=1, \cdots, 4, \\
& \gamma_{1}-\operatorname{Trace}(P) \geq 0 .
\end{aligned}
$$

On the other hand, we can minimize the size of the attractor $\mathcal{A}$ via the optimization problem as below

$$
\begin{aligned}
& \max _{\gamma_{2}, P, P_{a}, \tau_{1}, \cdots, \tau_{4}} \gamma_{2}, \quad \text { subject to }(31)-(36) \text { and } \\
& \gamma_{2}>0, \quad P>0, \quad \tau_{i}>0, i=1, \cdots, 4, \\
& \operatorname{Trace}\left(P_{a}\right)-\gamma_{2} \geq 0
\end{aligned}
$$

It may often be desirable to jointly optimize the size of the sets $\mathcal{D}$ and $\mathcal{A}$. This joint optimization is, in general, a difficult problem. A way to jointly achieve $\mathcal{D}$ of a large size and $\mathcal{A}$ of a small size is to minimize $\gamma:=\gamma_{1} / \gamma_{2}$, where $\gamma_{1}$ and $\gamma_{2}$ are the parameters in (38) and (39). This optimization problem can be formulated as follows. First, define

$$
\kappa=\gamma_{2}^{-1}, X=\kappa P, X_{a}=\kappa P_{a}, \alpha_{i}=\kappa \tau_{i}, i=1,2,4, \alpha_{3}=\tau_{3}
$$

where $P, P_{a}, \tau_{1}, \cdots, \tau_{4}$ are as in (31)-(36). Multiplying (31)-(36), (38) and (39) by $\kappa$, these inequalities become

$$
\begin{gathered}
\gamma-\operatorname{Trace}(X) \geq 0 \\
\operatorname{Trace}\left(X_{a}\right)-1 \geq 0 \\
X_{a}-X>0 \\
X-\kappa(1-\delta)^{2} \mu^{-2} C_{i}^{\prime} C_{i}>0
\end{gathered}
$$




$$
\begin{gathered}
{\left[\begin{array}{cc}
A_{i}^{\prime} X A_{i}-X-\alpha_{1}\left(1-\delta^{2}\right) C_{i}^{\prime} C_{i} & A_{i}^{\prime} X B_{i}+\alpha_{1} C_{i}^{\prime} \\
B_{i}^{\prime} X A_{i}+\alpha_{1} C_{i} & B_{i}^{\prime} X B_{i}-\alpha_{1}
\end{array}\right]<0} \\
{\left[\begin{array}{cc}
A_{i}^{\prime} X_{a} A_{i}-X_{a}-\alpha_{2}\left(1-\delta^{2}\right) C_{i}^{\prime} C_{i} & A_{i}^{\prime} X_{a} B_{i}+\alpha_{2} C_{i}^{\prime} \\
B_{i}^{\prime} X_{a} A_{i}+\alpha_{2} C_{i} & B_{i}^{\prime} X_{a} B_{i}-\alpha_{2}
\end{array}\right]<0} \\
\alpha_{3} \kappa-\alpha_{4} \geq 0 \\
X_{a}-\left(1+\alpha_{3}\right) A_{i}^{\prime} X_{a} A_{i}+\alpha_{4} \varepsilon^{-2} C_{i}^{\prime} C_{i} \geq 0
\end{gathered}
$$

Then, the optimization problem is as follows:

$$
\left\{\begin{array}{c}
\min _{\gamma, \kappa, X, X_{a}, \alpha_{1}, \cdots, \alpha_{4}} \gamma, \quad \text { subject to (40)-(47) and } \\
\kappa>0, \quad X>0, \quad \alpha_{i}>0, i=1, \cdots, 4
\end{array}\right.
$$

and we have that $P=\kappa^{-1} X$ and $P_{a}=\kappa^{-1} X_{a}$.

Notice that remarks similar to those of Remark 4.2 apply to the three latter optimization problems.

\section{Finite-Level Quantizer Construction}

Theorem 4.1 provides a method of deriving a set of admissible initial states $\mathcal{D}$ and its attractor $\mathcal{A}$ for a finite-level quantizer with given maximum quantization level $\mu$ and zerolevel error $\varepsilon$. However, this theorem can be applied to design a quantizer which guarantees wide quadratic stability. Given the set $\mathcal{D}_{0}=\left\{\zeta: \zeta^{\prime} P_{0} \zeta \leq 1\right\}, P_{0}>0$, of admissible initial states and an upper-bound $\vartheta$ of the volume of an attractor $\mathcal{A}=\left\{\zeta: \zeta^{\prime} P_{a} \zeta \leq 1\right\}^{1}$ of $\mathcal{D}_{0}$, with $P_{a}>0$ to be found, a quantizer $Q(\cdot)$ can be obtained by the following procedure:

Step 1: Design a controller via Theorem 3.1 or 3.2 and choose the quantization density $\rho$ of $Q(\cdot)$ such that $\rho \geq \rho_{\text {inf }}$.

Step 2: Find matrices $P>0, P_{a}>0$ and positive scalars $\gamma_{1}$, $\gamma_{2}, \tau_{1}, \cdots, \tau_{4}$ satisfying (32) with $\mu^{-2}=\gamma_{1}$, (33), (34) and

$$
\begin{gathered}
P_{0}-P>0, \quad P_{a}-P_{0}>0, \\
\tau_{3} \gamma_{2}-\tau_{4} \geq 0, \\
P_{a}-\left(1+\tau_{3}\right) A_{i}^{\prime} P_{a} A_{i}+\tau_{4} C_{i}^{\prime} C_{i} \geq 0, \\
\vartheta^{\frac{2}{n_{i}}} P_{a}-\sigma^{\frac{2}{n_{i}}} I \geq 0 .
\end{gathered}
$$

Then, $\mu$ and $\varepsilon$ are given by $\mu=1 / \sqrt{\gamma_{1}}$ and $\varepsilon=1 / \sqrt{\gamma_{2}}$.

Step 3: The number of positive quantization levels is given by the smallest integer $N$ satisfying $N \geq 1+\log _{\rho} \frac{\varepsilon(1+\delta)}{\mu}$.

Notice that the inequalities of Step 2 are equivalent to (32)-(36) with extra conditions to ensure $\mathcal{D}_{0} \subset \mathcal{D}, \mathcal{A} \subset \mathcal{D}_{0}$ and the upper-bound $\vartheta$ for the volume of $\mathcal{A}$.

Similarly to Theorem 3.1 , we can either minimize $\mu$ or maximize $\varepsilon$ by solving optimization problems for minimizing $\gamma_{1}$ or maximizing $\gamma_{2}$ respectively, subject to the inequalities of Step 2. Moreover, we can jointly achieve a small $\mu$ and a large $\varepsilon$ by minimizing $\gamma:=\mu^{2} / \varepsilon^{2}$. This optimization problem can be readily derived by multiplying the inequalities of Step 2 by $\kappa:=\mu^{2}$ and setting $X=\kappa P, X_{a}=\kappa P_{a}, \alpha_{i}=\kappa \tau_{i}$, $i=1,2,4$, and $\alpha_{3}=\tau_{3}$, leading to

\footnotetext{
${ }^{1}$ The volume of $\mathcal{A}$ is given by $\sigma \prod_{k=1}^{n_{i}} \lambda_{k}^{-\frac{1}{2}}\left(P_{a}\right)$, where $\sigma$ is a constant that depends only on $n_{i}$ and $\lambda_{k}\left(P_{a}\right)$ are the eigenvalues of $P_{a}$.
}

$$
\begin{aligned}
& \min _{\gamma, \kappa, X, X_{a}, \alpha_{1}, \cdots, \alpha_{4}} \gamma, \text { subject to (44), (45), and } \\
& \kappa>0, \quad X>0, \quad \alpha_{i}>0, i=1, \cdots, 4 \\
& X-(1-\delta)^{2} C_{i}^{\prime} C_{i}>0 \text {, } \\
& \kappa P_{0}-X>0, \quad X_{a}-\kappa P_{0}>0, \\
& \alpha_{3} \gamma-\alpha_{4} \geq 0 \text {, } \\
& X_{a}-\left(1+\alpha_{3}\right) A_{i}^{\prime} X_{a} A_{i}+\alpha_{4} C_{i}^{\prime} C_{i} \geq 0 \text {, } \\
& \vartheta^{\frac{2}{n_{i}}} X_{a}-\sigma^{\frac{2}{n_{i}}} \kappa I \geq 0 \text {. }
\end{aligned}
$$

Moreover, we have $\mu=\sqrt{\kappa}, \varepsilon=\sqrt{\kappa / \gamma}, P=\kappa^{-1} X$ and $P_{a}=\kappa^{-1} X_{a}$.

Similarly to the optimization problems of Subsection IV$\mathrm{B}$, the latter optimization problem is nonconvex. However, for a given $\alpha_{3}$ the problem becomes convex. Thus, a way to minimize $\gamma$ via convex optimization is to search for the parameter $\alpha_{3}>0$ that achieves the smallest $\gamma$.

\section{Numerical Example}

Consider the non-minimum phase open-loop unstable discrete-time system of [11, Example 3.1] as given below

$$
\left\{\begin{aligned}
x_{1}(k+1) & =x_{2}(k) \\
x_{2}(k+1) & =2 x_{2}(x)+u(k) \\
y(k) & =-3 x_{1}(k)+x_{2}(k)
\end{aligned}\right.
$$

which has the transfer function $G(z)=\frac{z-3}{z(z-2)}$.

The controller is designed considering a quantizer with an infinite number of quantization levels. Applying Theorem 3.1 we obtain the following state feedback controller

$$
K=-\left[\begin{array}{ll}
0 & 1.99
\end{array}\right], \quad \rho_{\text {inf }}=1 / 3
$$

whereas with Theorem 3.2 we get an output feedback controller with

$$
A_{c}=-5, B_{c}=1, C_{c}=-50 / 3, D_{c}=10 / 3, \rho_{\mathrm{inf}}=0.83 .
$$

We assume that the finite-level quantizer has a maximum level $\mu=2.1$ for state feedback and output feedback, for both configurations I and II, and $\rho=\rho_{\text {inf }}$. Also, the maximum admissible zero-level error is chosen such that the conditions of Theorem 4.1 are satisfied.

For the above state feedback controller, Fig. 2 shows part of the set $\mathcal{D}$ of admissible initial states and its attractor $\mathcal{A}$ as obtained from the optimization problem in (48) along with a stable and two unstable state trajectories. The maximum admissible zero quantization level error is $\varepsilon=0.5$, yielding $N=2$ and thus the required number of bits $N_{b}$ for the quantizer is $N_{b}=3$.

Considering the above output feedback controller with a quantized measurement, i.e. in Configuration I, we obtain the results in Fig. 3, which displays a slice of $\mathcal{D}$ and $\mathcal{A}$ with $\xi=0$, as well as a stable and two diverging trajectories of the system state. Note that the maximum $\varepsilon$ for the LMIs of Theorem 4.1 to be feasible is $10^{-4}$, yielding $N=57$, which requires a quantizer with $N_{b}=7$.

Next, applying the output feedback controller with a quantized control signal, i.e. in Configuration II, leads to 
the results in Fig. 4, which shows a slice of $\mathcal{D}$ and $\mathcal{A}$ with $\xi=0$, together with a stable and two unstable trajectories of the system state. In this case, the maximum admissible $\varepsilon$ is $10^{-3}$, resulting in $N=44$ and $N_{b}=7$.

\section{CONCLUSION}

This paper has addressed the stability of discrete-time SISO linear time-invariant systems with a finite-level logarithmically quantized feedback controller. Both state and output feedback controllers have been considered. Based on a relaxed stability notion, referred to as wide quadratic stability, we have developed an LMI based approach to estimate a set of admissible initial states and an associated invariant attractor set in a neighborhood of the origin, such that all state trajectories starting in the first set will converge to the attractor in finite time. In addition, when these two sets are a priori specified, we have proposed a method to design either a state feedback controller or an output feedback controller and a finite-level logarithmic quantizer which ensure wide quadratic stability. A numerical example has shown that for state feedback, wide quadratic stability can be guaranteed with a relatively small number of bits, contrasting with the output feedback case in which the number of bits is significantly larger. In addition, the size of set of admissible initial states in the output feedback setting is much smaller when compared with the state feedback case.

\section{REFERENCES}

[1] R. E. Kalman, "Nonlinear aspects of sampled-data control systems," in Proc. Symp. Nonlinear Circuit Theory, vol. VII, Brooklyn, NY, 1956.

[2] J. B. Slaughter, "Quantization errors in digital control systems," IEEE Trans. Autom. Control, vol. 9, pp. 70-74, 1964.

[3] D. F. Delchamps, "Stabilizing a linear system with quantized state feedback," IEEE Trans. Autom. Control, vol. 35, pp. 916-924, 1990.

[4] W. S. Wong and R. W. Brockett, "Systems with finite communication bandwidth constraints II: Stabilization with limited information feedback," IEEE Trans. Autom. Control, vol. 44, pp. 1049-1053,1999.

[5] R. W. Brockett and D. Liberzon, "Quantized feedback stabilization of linear systems," IEEE Trans. Autom. Control, vol. 45, pp. 1279-1289, July 2000 .

[6] G. N. Nair and R. J. Evans, "Stabilization with data-rate-limited feedback: tightest attainable bounds," Systems \& Control Letts., vol. 41, pp. 49-56, 2000.

[7] N. Elia and S. K. Mitter, "Stabilization of linear systems with limited information," IEEE Trans. Autom. Control, vol. 46, pp. 1384-1400, 2001.

[8] G. N. Nair and R. J. Evans, "Exponential stabilization of multidimensional linear systems," Automatica, vol. 39, pp. 585-593, 2003.

[9] H. Ishii and B. A. Francis, "Quadratic stabilization of sampled-data systems with quantization," Automatica, vol. 39, pp. 1793-1800, 2003.

[10] S. Tatikonda and S. K. Mitter, "Control under communications constraints," IEEE Trans. Autom. Control, vol. 49, pp. 1056-1068, 2004.

[11] M. Fu and L. Xie, "The sector bound approach to quantized feedback control," IEEE Trans. Autom. Control, vol. 50, pp. 1698-1711, 2005.

[12] M. Fu and L. Xie, "Finite-level quantized feedback control for linear systems," in Proc. IEEE Conf. Decision and Control, pp. 1117-1122, San Diego, CA, Dec. 2006.

[13] D.F. Coutinho, M. Fu and C.E. de Souza, "Quantized feedback control of linear systems with input and output quantization," in Proc. IEEE Conf. Decision and Control, Dec. 2008 (to appear).

[14] P. Gahinet, "Explicit controller formulas for LMI-based $H_{\infty}$ synthesis," Automatica, vol. 32, pp. 1007-1014, 1996.

[15] S. Boyd, L. El-Ghaoui, E. Feron and V. Balakrishnan, Linear Matrix Inequalities in System and Control Theory, SIAM, 1994.

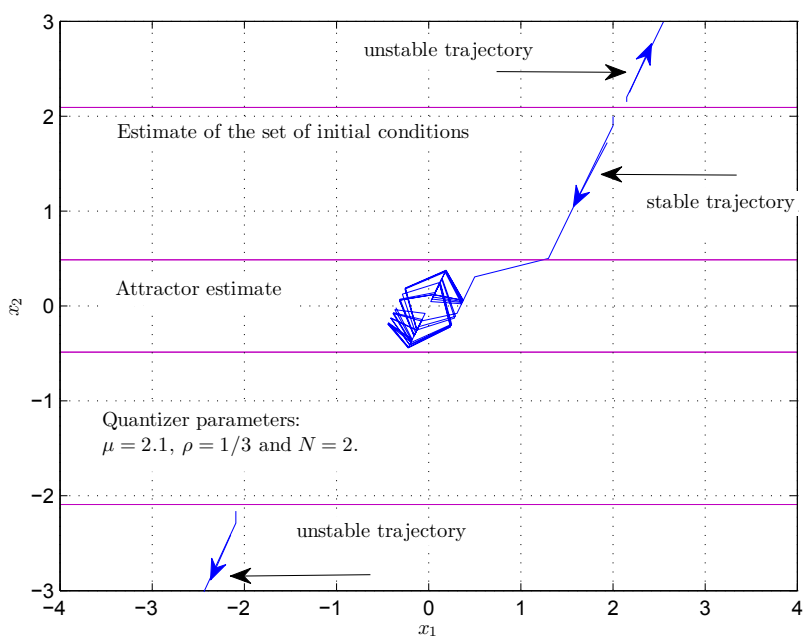

Fig. 2. Sets $\mathcal{D}$ and $\mathcal{A}$ for the state feedback controller, and a stable and two unstable state trajectories.

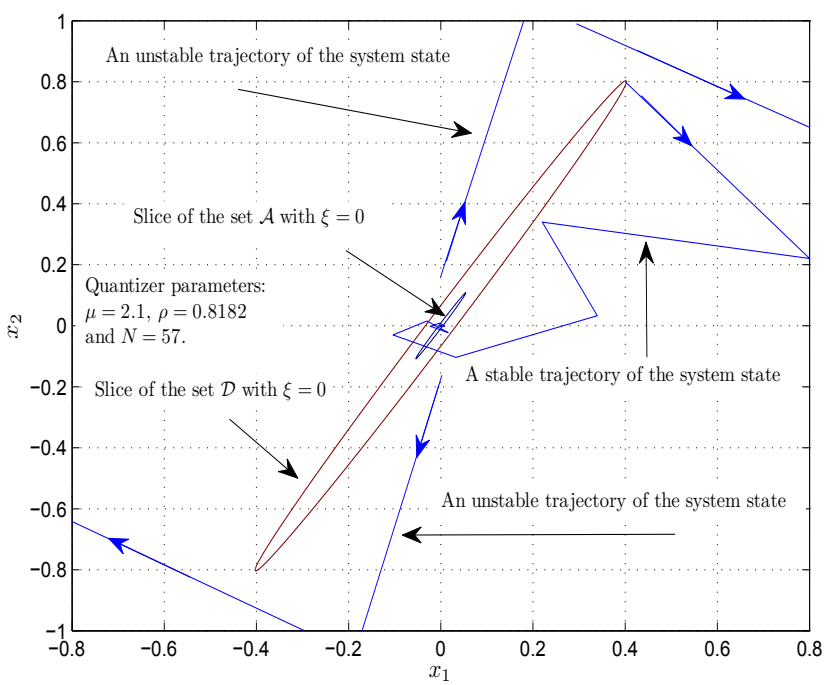

Fig. 3. A slice of the sets $\mathcal{D}$ and $\mathcal{A}$ (with $\xi=0$ ) for output feedback in Configuration I, and a stable and two unstable state trajectories.

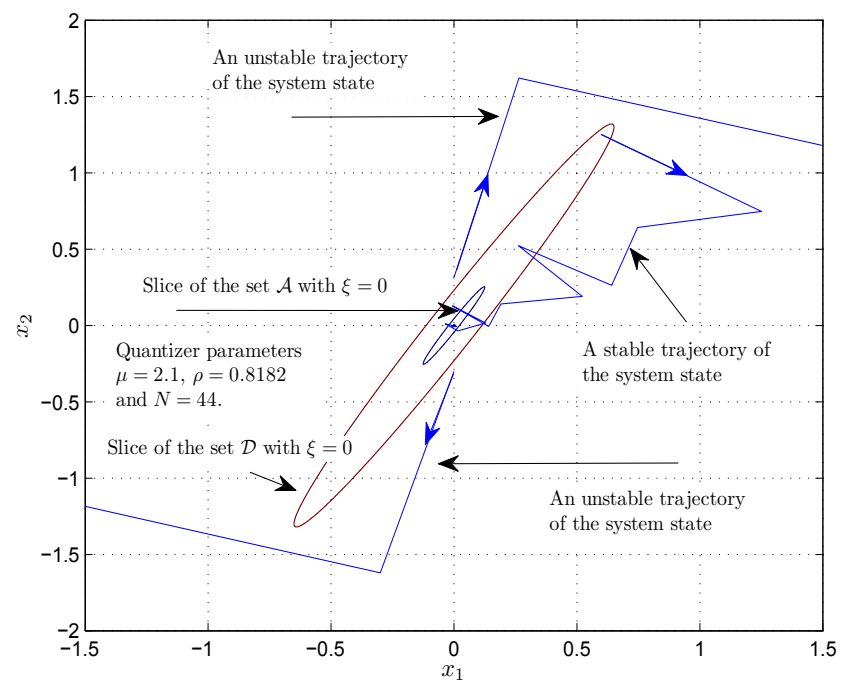

Fig. 4. A slice of the sets $\mathcal{D}$ and $\mathcal{A}$ (with $\xi=0$ ) for output feedback in Configuration II, and a stable and two unstable state trajectories. 